\title{
Increased blood culture sensitivity in pediatric oncology patients: is it the peripheral culture or increased collected blood volume?
}

\author{
Thomas V. Adamkiewicz
}

Received: 4 December 2009 /Accepted: 29 March 2010/Published online: 16 May 2010

(C) Springer-Verlag 2010

Scheinemann et al. [3] re-examined retrospectively the occurrence of central vs. peripheral blood cultures on the same oncology ward where a similar study was conducted previously [1]. The principal observation from the original study was that peripheral blood cultures were seldom clinically useful as a means to identify central line infections. However, both studies showed that the addition of a peripheral culture overall improves sensitivity to detect true pathogens. As Scheinemann et al. pointed out, it is possible that the same could have been achieved by drawing a larger blood draw from a single site. Indeed, in a recent study, pediatric blood cultures with measured volume $\geq 4 \mathrm{ml}$ in children $\geq 36$ months yielded significantly more positive results [2]. Scheinemann et al. defined 1 to $3 \mathrm{ml}$ as an adequate collection volume, at time of collection. Volumes were not confirmed after collection. Adding a peripheral culture would have doubled the total volume collected and increased the likelihood of recovering a pathogen. Also, peripheral blood cultures contributed to the cumulative likelihood of isolating contaminants, thus the lowering of overall specificity. In the context where optimal blood volumes are collected from central lines, the utility of peripheral blood cultures still can be questioned. A welldesigned prospective study to help determine best blood culture collection practices in pediatric oncology patients is needed. Effects of volume, timing (single vs. tandem draw), and site (central vs. peripheral) would be examined separately. Such a study should include sampling before and after start of antibiotic therapy, as positive culture rates remain significant after antibiotics are started [1], and presence of antibiotics may change culture recovery conditions. Such a study could include repeated central line cultures as control, an approach that could also help identify contaminants.

\section{References}

1. Adamkiewicz TV, Lorenzana A, Doyle J, Richardson S (1999) Peripheral vs. central blood cultures in patients admitted to a pediatric oncology ward. Pediatr Infect Dis J 18(6):556-558

2. Connell TG, Rele M, Cowley D, Buttery JP, Curtis N (2007) How reliable is a negative blood culture result? Volume of blood submitted for culture in routine practice in a children's hospital. Pediatrics 119(5):891-896

3. Scheinemann K, Ethier MC, Dupuis LL, Richardson SE, Doyle J, Allen U, Sung L (2009) Utility of peripheral blood cultures in bacteremic pediatric cancer patients with a central line. Support Care Cancer. doi:10.1007/s00520-009-0725-0

T. V. Adamkiewicz $(\square)$

Co-PI MSM T90/R90 Training Program in Genomics and Hemoglobinopathies, Department of Family Medicine, Morehouse School of Medicine,

1513 E Cleveland Ave, Building 100, Suite 300-A,

East Point, GA 30344, USA

e-mail: tadamkiewicz@msm.edu 